\title{
Acute Cholecystitis: Computed Tomography (CT) versus Ultrasound (US)
}

\author{
Ee Syn Tan'1, Jason Friesen², Brendon Friesen ${ }^{3 *}$ \\ ${ }^{1}$ Department of General Surgery, Monash Health, Dandenong Hospital, Dandenong, Australia \\ ${ }^{2}$ Royal Brisbane and Women's Hospital, University of Queensland, Brisbane, Australia \\ ${ }^{3}$ Lake Imaging, St. John of God, Geelong, Australia \\ Email: eesyn.tan@monashhealth.org, jason_friesen@live.com, ^friesenb@lakeimaging.com.au
}

How to cite this paper: Tan, E.S., Friesen, J. and Friesen, B. (2018) Acute Cholecystitis: Computed Tomography (CT) versus Ultrasound (US). Open Journal of Radiology, 8, 131-139.

\section{https://doi.org/10.4236/ojrad.2018.82015}

Received: May 22, 2018

Accepted: June 25, 2018

Published: June 28, 2018

Copyright (C) 2018 by authors and Scientific Research Publishing Inc. This work is licensed under the Creative Commons Attribution International License (CC BY 4.0).

http://creativecommons.org/licenses/by/4.0/

\section{cc) (i) Open Access}

\begin{abstract}
Acute cholecystitis is a common surgical emergency and ultrasound (US) is currently considered the first-line diagnostic imaging test. The relative accuracy of computed tomography (CT) in detecting acute cholecystitis has received little attention in the literature. We report a case series of 113 patients who underwent emergency laparoscopic cholecystectomy at a tertiary surgical centre in Australia, between 2014 and 2016, after undergoing both US and CT examination for acute right upper quadrant pain. Both US and CT had a relatively low sensitivity in detecting acute cholecystitis in the patients with histologically proven acute cholecystitis ( $47 \%$ and $45 \%$ respectively) but high specificity ( $84 \%$ and $79 \%$ respectively). As expected, US was much more sensitive in detecting cholelithiasis (92\%) in comparison to CT (55\%). With the added advantage of CT in excluding other alternative intra-abdominal pathology in patients presenting with acute right upper quadrant pain, and similar accuracy in detecting acute cholecystitis, the need for ultrasound may be negated in cases where acute cholecystitis has been confirmed on CT. However, it is noted that both US and CT had a significant false negative rate for acute cholecystitis, and if there remains a clinical suspicion despite initial normal imaging, repeat delayed imaging and/or surgical opinion may be warranted.
\end{abstract}

\section{Keywords}

Acute Cholecystitis, Ultrasound, Computed Tomography, Gallbladder Disease

\section{Introduction}

Acute cholecystitis is a common surgical emergency and is defined as inflammation of the gallbladder secondary to an obstruction at the gallbladder neck, often 
due to cholelithiasis [1]. In Australia, 380,000 patient-doctor encounters occur nationwide on an annual basis related to gallbladder disease [2]. Early diagnosis of acute cholecystitis, prompting early surgical intervention, leads to better patient outcomes post-laparoscopic cholecystectomy [3].

Ultrasound (US) is the preferred initial imaging modality for biliary tract disease including suspected cholecystitis [4]. It has a greater sensitivity and specificity than CT or MRI. Greater diagnostic accuracy has been observed with cholescintigraphy [5], however due to clinician preference and cost, ultrasound is preferred. US features of acute cholecystitis include gallbladder probe tenderness (or sonographic Murphy sign), gallbladder wall thickening ( $3 \mathrm{~mm}$ or greater), presence of gallstones impacted in the gallbladder neck, gallbladder luminal distension, gallbladder wall hyperaemia and pericholecystic fluid [6]. In the literature, the specificity of US has been reported to be as high as $95 \%-99 \%$ in acute calculous cholecystitis, with sensitivities ranging from $84 \%$ - 97\% [5]. US is highly sensitive in diagnosing cholelithiasis and is radiation free. However, it is limited by the patient's body habitus, potential obscuring bowel gas, the requirement for patients to fast for at least 6 to 8 hours prior to scanning and the availability of equipment and trained sonographers after-hours [7].

Computed tomography (CT) is an alternative or complementary test in the assessment of acute cholecystitis despite its reported low sensitivity in detecting cholelithiasis in the literature. Sensitivity and specificity of CT in diagnosing acute cholecystitis is an under-evaluated area in the literature [8]. CT features of acute cholecystitis include pericholecystic inflammatory change (or fat stranding), gallbladder wall thickening, pericholecystic fluid, gallbladder wall enhancement or presence of intramural gas [9]. There is increasing utilisation of CT because of its ready availability after hours, newer scanners and reconstruction algorithms with decreasing radiation dose and scanning time, and ability to exclude alternative intra-abdominal pathology such as diverticulitis.

We report a case series of patients undergoing emergency laparoscopic cholecystectomy from 2014 to 2016 in a large tertiary institution after undergoing both diagnostic US and CT examination. The primary aim of this study is to investigate the relative accuracy of CT and US in the diagnosis of acute cholecystitis, which is of interest to emergency physicians, surgeons and radiologists.

\section{Method}

A retrospective search of the surgical database was performed on patients undergoing emergency laparoscopic cholecystectomy over a 48-month period at our institution dating from 1 January 2014 to 1 January 2016. An appropriate sample size was determined using $\mathrm{G}^{*}$ Power 3.1 software. Patients requiring emergent cholecystectomy who had both US and CT within 72 to 96 hours prior to their surgical procedure were included in the study. Correlative imaging and pathology reports were reviewed. Patients undergoing outpatient cholecystectomy and paediatric patients were excluded from the study. 


\section{Selection criteria:}

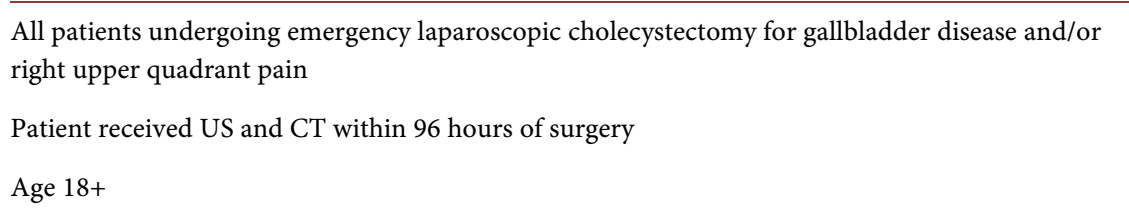

CT diagnosis of acute cholecystitis was based on the combination of gallbladder wall thickening, pericholecystic inflammatory change or fluid and gallbladder luminal distension. The presence and absence of cholelithiasis is noted but not criteria for diagnosis of acute cholecystitis. Diagnostic features of US indicating acute cholecystitis were based on the presence of at least gallbladder wall thickening of $3 \mathrm{~mm}$ or greater and sonographic probe tenderness with or without cholelithiasis.

Histopathological diagnosis was considered as the reference standard for diagnosis of acute cholecystitis. Positive results were recorded if the reporting pathologists had noted acute changes on the tissue specimen analysis. This often consists of neutrophil infiltration or presence of abscess formation. Serum neutrophil count prior to surgical intervention is documented for each patient as well. To determine the relative accuracy of US and CT in the diagnosis of acute cholecystitis, patient radiology reports and images were reviewed and compared to histopathological results. For both US and CT, results were characterised as either false positive, true positive, false negative or false positive. Using this data, sensitivities, specificities and odds ratios were calculated. McNemar's test was used to analyse the difference between the two imaging modalities. Patient data was de-identified and stored securely.

\section{Results}

There were 113 patients included in this study with a mean age of 59 years (range 29 - 90 years). There were 58 men and 55 women in this case series. All histopathological specimens demonstrated inflammatory changes in keeping with chronic cholecystitis. There were no patients with a "normal" gallbladder on histology. 49 cases had evidence of acute-on-chronic cholecystitis on histology. Out of these cases, a small proportion (17 cases) showed acute cholecystitis in the absence of cholelithiasis (acalculous cholecystitis). There were 35 cases diagnosed with acute cholecystitis on both CT and US.

\begin{tabular}{llc}
\hline \multicolumn{3}{c}{113 patients } \\
\hline 58 male & 55 female \\
& Ages $29-90$ & \\
\hline
\end{tabular}

Sensitivity of CT in detecting acute cholecystitis was $45 \%$ and specificity $79 \%$. False positive and false negative rates were $21 \%$ and $55 \%$ respectively for CT. 
The sensitivity and specificity for US in diagnosing acute cholecystitis was $47 \%$ and $84 \%$ respectively. False positive and false negative rates for US were $16 \%$ and $53 \%$ respectively (see Table 1 ). McNemar's test analysis performed on the data showed no significant difference between these two imaging modalities ( $\mathrm{p}=$ 0.71). In detecting cholelithiasis, the sensitivity of US was $92 \%$ and the specificity was $55 \%$. For CT, the sensitivity and specificity for detecting cholelithiasis was $56 \%$ and $36 \%$ respectively (see Table 2 ). For acute acalculous cholecystitis, the sensitivity and specificity of US was $31 \%$ and $95 \%$ respectively and for CT, the sensitivity and specificity was $50 \%$ and $80 \%$ respectively (see Table 3 ). The relatively small sample of acalculous cholecystitis patients limited a reliable direct comparison in the diagnostic accuracy of CT and US when compared against the larger acute cholecystitis group.

Several statistically significant figures were obtained when each imaging parameter were analysed. Even when the sensitivity of both US and CT were relatively low in detecting acute cholecystitis, the odds ratio of CT and US in detecting acute cholecystitis is $3(\mathrm{p}<0.01)$ and 4.6 ( $\mathrm{p}<0.01)$ respectively. Gallbladder wall thickening and sonographic probe tenderness were reliable predictors of acute cholecystitis as the odds ratio for gallbladder wall thickening $(3 \mathrm{~mm}$ or greater) was 6.27 (95\% CI 2.72 - 14.45) and for sonographic probe tenderness an odds ratio of 4.53 (95\% CI 1.94 - 10.57). CT findings of pericholecystic fluid were a good indicator for acute cholecystitis with an odds ratio of 3.86 (95\% CI 1.55 - 9.62) (see Figure 1).

\section{Discussion}

There are not many comparative studies between CT and US in the diagnosis of acute cholecystitis [10]. In our series, the sensitivities for US and CT were relatively

Factor-Factor +

Neutrophil count $(<5 />5)$
US ACC (No/Yes)
US Cholelithiasis $(\mathrm{No} / Y e s)$
US Polyps (No/Yes)
US GB Wall thickness $(<3 \mathrm{~mm} />3 \mathrm{~mm})$
US Probe tender $(\mathrm{No} / \mathrm{Yes})$
CT ACC $(\mathrm{No} / \mathrm{Yes})$
CT Pericholecystic fluid $(\mathrm{No} / \mathrm{Yes})$
CT GB Distension $(\mathrm{No} / \mathrm{Yes})$
CT Cholelithiasis $(\mathrm{No} / \mathrm{Yes})$

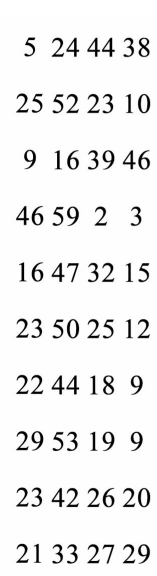

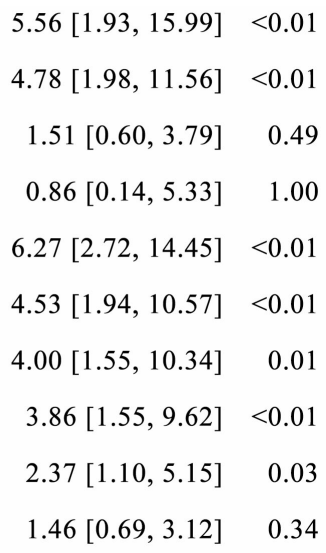

Figure 1. Odds ratio for each imaging parameter in detecting acute cholecystitis. 
Table 1. Demonstrates the number of cases positive and negative for acute cholecystitis on CT, US and histology.

\begin{tabular}{ccccc}
\hline & $\begin{array}{c}\text { Acute cholecystitis } \\
\text { on US }\end{array}$ & $\begin{array}{c}\text { No acute } \\
\text { cholecystitis on US }\end{array}$ & $\begin{array}{c}\text { Acute } \\
\text { cholecystitis on } \\
\text { CT }\end{array}$ & $\begin{array}{c}\text { No acute } \\
\text { cholecystitis on } \\
\text { CT }\end{array}$ \\
\hline $\begin{array}{c}\text { Positive histology } \\
\text { (acute cholecystitis) }\end{array}$ & 23 & 26 & 22 & 27 \\
$\begin{array}{c}\text { Negative histology } \\
\text { (chronic cholecystitis } \\
\text { without acute changes) }\end{array}$ & 10 & 52 & 13 & 48 \\
\hline
\end{tabular}

Table 2. Demonstrates the proportion of cases that had cholethiasis detected on CT, US and histology.

\begin{tabular}{lcccc}
\hline & $\begin{array}{c}\text { Cholelithiasis on } \\
\text { US }\end{array}$ & $\begin{array}{c}\text { No cholelithiasis on } \\
\text { US }\end{array}$ & $\begin{array}{c}\text { Cholelithiasis on } \\
\text { CT }\end{array}$ & $\begin{array}{c}\text { No cholelithiasis } \\
\text { on CT }\end{array}$ \\
\hline $\begin{array}{c}\text { Cholelithiasis in } \\
\text { histology }\end{array}$ & 69 & 6 & 42 & 33 \\
$\begin{array}{c}\text { No cholelithiasis in } \\
\text { histology }\end{array}$ & 16 & 20 & 14 & 22 \\
\hline
\end{tabular}

Table 3. Demonstrates the proportion of cases with acute acalculous cholecystitis on CT, US and histology.

\begin{tabular}{|c|c|c|c|c|}
\hline & $\begin{array}{l}\text { Acute acalculous } \\
\text { cholecystitis on US }\end{array}$ & $\begin{array}{l}\text { No acute acalculous } \\
\text { cholecystitis on US }\end{array}$ & $\begin{array}{l}\text { Acute acalculous } \\
\text { cholecystitis on CT }\end{array}$ & $\begin{array}{c}\text { No acute } \\
\text { acalculous } \\
\text { cholecystitis on } \\
\text { CT }\end{array}$ \\
\hline $\begin{array}{l}\text { Histology showing } \\
\text { acute acalculous } \\
\text { cholecystitis }\end{array}$ & 5 & 11 & 8 & 8 \\
\hline $\begin{array}{c}\text { Negative histology } \\
\text { for acute acalculous } \\
\text { cholecystitis }\end{array}$ & 1 & 19 & 3 & 17 \\
\hline
\end{tabular}

low ( $47 \%$ and $45 \%$ respectively) in comparison to recent literature where sensitivity of US in detecting cholecystitis ranges between $73 \%$ to $100 \%$ and for CT a sensitivity of $73 \%$ [5] [11]. However, one study did report a sensitivity of 54\% and specificity of $81 \%$ for acute cholecystitis on ultrasound [12] and another reported a higher sensitivity of $92 \%$ in CT in the detection of acute cholecystitis when compared to US (79\%) [13]. It should be noted that there are other reliable modalities in detecting acute cholecystitis such as radionuclide scanning and magnetic resonance imaging but their utilisation is limited in the acute setting [6].

Laparoscopic cholecystectomy is a standard surgical procedure offered to patients with acute calculous cholecystitis and biliary colic. Nevertheless, surgical intervention is sometimes required for acalculous cholecystitis [14]. Acalculous cholecystitis can develop in the setting of severe systemic disease, diabetes mellitus, malignant disease, vasculitis, congestive cardiac disease or shock [15]. In our case series, there were 17 patients with acute acalculous cholecystitis and the 
sensitivity of US and CT in detecting this is moderately low but highly specific. We acknowledge the inherent bias of our case series, such that the patients were drawn from a cohort of all patients undergoing emergent cholecystectomy, rather than a cohort presenting with acute right upper quadrant pain, or those with cholecystitis managed non-operatively. This is a limitation of the study design. Other limitations of this study include the fact that diagnosis made by CT and US involves subjective assessment. Wall thickening is subject to observer variability. Presence or absence of pericholecystic inflammatory change is a subjective assessment. Assessment of gallbladder probe tenderness on US is subjective, and may be influenced by administration of opioid analgesia prior to US. There is a possibility that a proportion of the acalculous cholecystitis group had gallstones that were missed on both imaging and during surgery. Small cystic duct calculi may have gone undetected for example.

As expected in our case series, US remains the superior tool to CT in detecting cholelithiasis (see Figure 2), consistent with the literature [16]. CT detection of cholelithiasis relies heavily on the presence of calcification and occasionally fat or gas attenuation (see Figure 3) with many gallstones being occult on CT. The specificity of CT in detecting cholelithiasis in this study is $55 \%$ which is comparable to the literature [13].

Our study suggests that certain parameters are highly associated with acute cholecystitis such as gallbladder wall thickening (3 $\mathrm{mm}$ or greater) and sonographic probe tenderness (see Figure 4) but careful consideration should be

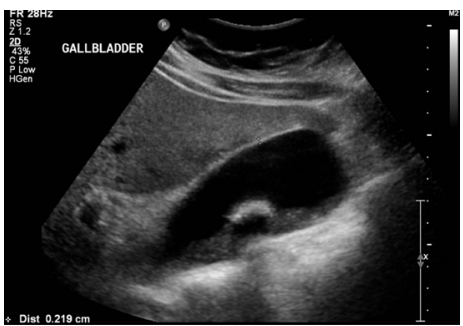

(a)

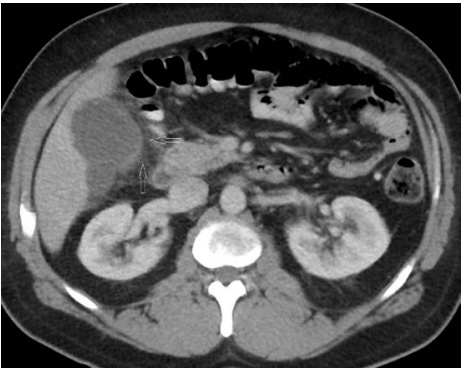

(c)

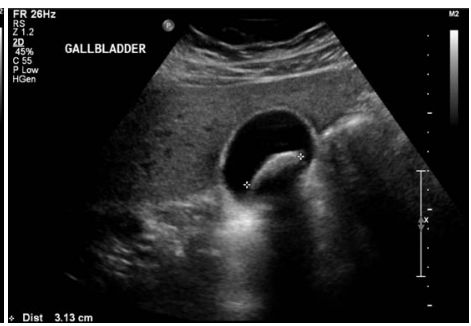

(b)

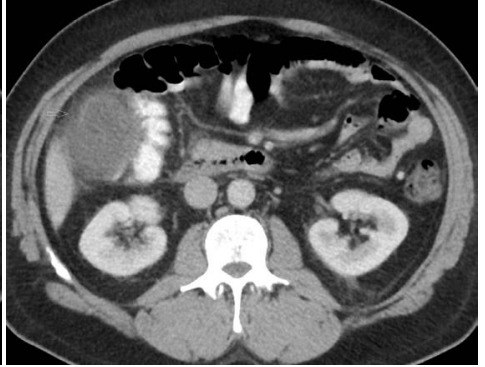

(d)

Figure 2. 47-year-old man with acute calculous cholecystitis (histologically proven). Longitudinal (a) and transverse (b) ultrasound of the gallbladder. A single $3 \mathrm{~cm}$ mobile gallstone is noted. There is no appreciable gallbladder wall thickening or probe tenderness. Portal venous phase CT abdomen and pelvis (performed approximately 48 hours later) demonstrates pericholecystic fat stranding (arrows in (c) and (d)) and patchy gallbladder wall hyper-enhancement. The gallstone seen on ultrasound is not appreciable. 


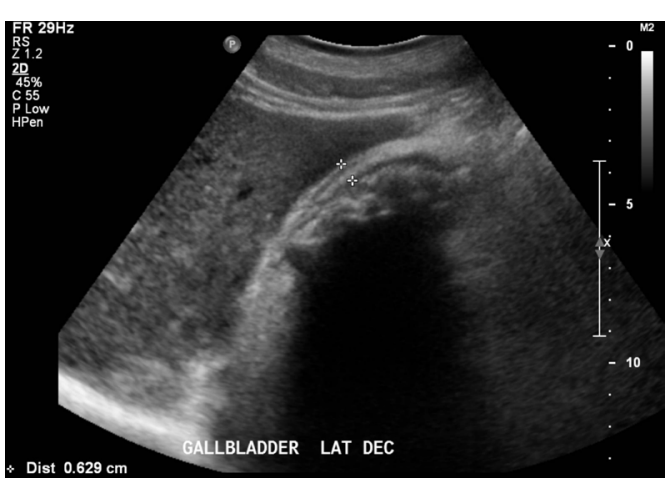

(a)

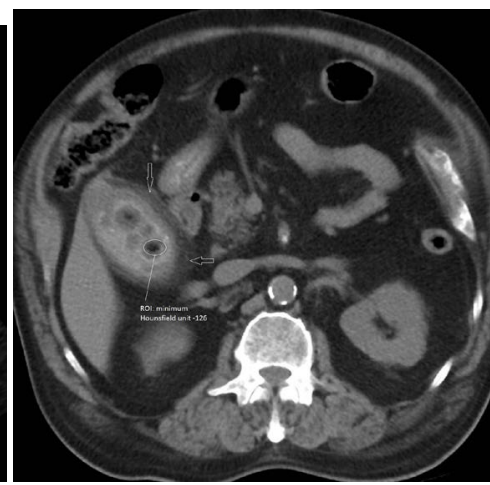

(b)

Figure 3. 80-year-old man with acute calculous cholecystitis (histologically proven). Ultrasound (a) demonstrates gallbladder wall thickening $(6 \mathrm{~mm})$; gallbladder lumen is filled with calculi with posterior shadowing and positive sonographic Murphy sign. Non-IV contrast CT abdomen ((b), axial) demonstrates multiple gallstones with central low attenuation (Hounsfield unit of minus 126), compatible with fat or gas content. There is gallbladder wall thickening and pericholecystic inflammatory change (see arrows).

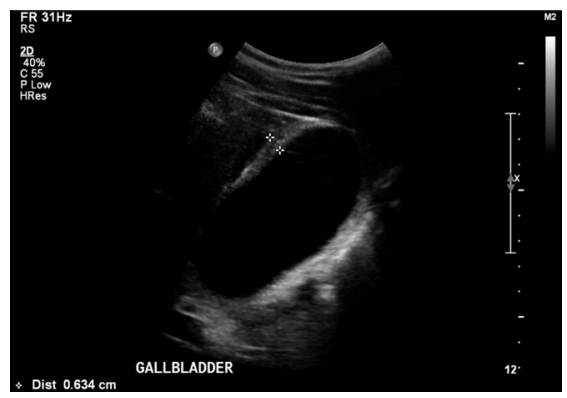

(a)

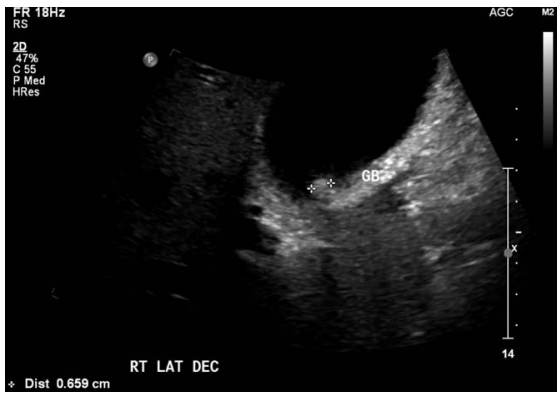

(b)

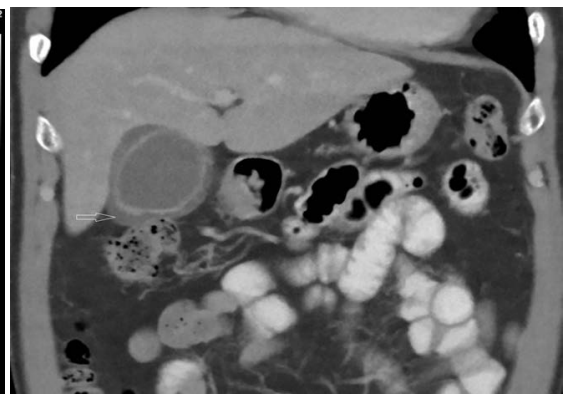

(c)

Figure 4. 63-year-old man with acute calculous cholecystitis (histologically proven). Ultrasound (a) (b) demonstrates gallbladder wall thickening $(6 \mathrm{~mm})$, a mobile $7 \mathrm{~mm}$ gallstone, trace pericholecystic fluid and positive sonographic Murphy sign. Portal venous phase CT abdomen (c), coronal reconstruction) demonstrates prominent gallbladder wall oedema, pericholecystic fluid (arrow) and gallbladder wall hyper-enhancement.

given to such findings due to confounding factors. Sonographic probe tenderness is a subjective finding and can be masked by the presence of analgesia prior to scanning. Gallbladder wall thickening can herald other medical conditions such as cardiac failure, liver failure, hypoalbuminaemia or hepatitis [17].

We report a significant false negative rate for both US and CT and one poten- 
tial reason for this finding is that the pathological appearance on imaging may be influenced by the length of time after onset of symptoms and time of scanning (refer to previous Figure 2).

\section{Conclusion}

We conclude that CT is comparable to US in the diagnosis of acute cholecystitis. The normal ultrasound and/or CT in the acute setting does not necessarily exclude acute cholecystitis, as both imaging modalities demonstrated a high false negative rate. Patients presenting to the emergency with clinical suspicion of acute cholecystitis but negative imaging may benefit from surgical opinion or in certain cases delayed imaging.

\section{References}

[1] Kimura, Y., Takada, T., Kawarada, Y., Nimura, Y., Hirata, K., Sekimoto, M., et al. (2007) Definitions, Pathophysiology, and Epidemiology of Acute Cholangitis and Cholecystitis: Tokyo Guidelines. Journal of Hepato-Biliary-Pancreatic Surgery, 14, 15-26. https://doi.org/10.1007/s00534-006-1152-y

[2] Bayram, C., Valenti, L. and Miller, G.C. (2013) Gallbladder Disease. Australian Family Physician, 42, 443.

[3] Zhou, M.W., Gu, X.D., Xiang, J.B. and Chen, Z.Y. (2014) Comparison of Clinical Safety and Outcomes of Early versus Delayed Laparoscopic Cholecystectomy for Acute Cholecystitis: A Meta-Analysis. The Scientific World Journal, 2014, Article ID: 274516. https://doi.org/10.1155/2014/274516

[4] Ansaloni, L., Pisano, M., Coccolini, F., Peitzmann, A.B., Fingerhut, A., Catena, F., et al. (2016) WSES Guidelines on Acute Calculous Cholecystitis. World Journal of Emergency Surgery, 11, 25. https://doi.org/10.1186/s13017-016-0082-5

[5] Shea, J.A., Berlin, J.A., Escarce, J.J., Clarke, J.R., Kinosian, B.P., Cabana, M.D., Tsai, W.W., et al. (1994) Revised Estimates of Diagnostic Test Sensitivity and Specificity in Suspected Biliary Tract Disease. Archives of Internal Medicine, 154, 2573-2581. https://doi.org/10.1001/archinte.1994.00420220069008

[6] Barie, P.S. and Eachempati, S.R. (2003) Acute Acalculous Cholecystitis. Current Gastroenterology Reports, 5, 302-309. https://doi.org/10.1007/s11894-003-0067-x

[7] Greiner, L. (2009) Clinical Abdominal Ultrasonography (US) -Benefits, Potentials, and Limitations. Medical Ultrasonography, 11, 33-36.

[8] Kiewiet, J.J.S., Leeuwenburgh, M.M.N., Bipat, S., Bossuyt, P.M.M., Stoker, J., Boermeester, M.A., et al. (2012) A Systemic Review and Meta-Analysis of Diagnostic Performance of Imaging in Acute Cholecystitis. Radiology, 264, 708-720.

https://doi.org/10.1148/radiol.12111561

[9] Patel, N.B., Oto, A. and Thomas, S. (2013) Multidetector CT of Emergent Biliary Pathologic Conditions. Radiographics, 33, No. 7. https://doi.org/10.1148/rg.337125038

[10] Mirvis, S.E., Whitley, N.O. and Miller, J.W. (1987) CT Diagnosis of Acalculous Cholecystitis. Journal of Computer Assisted Tomography, 11, 83-87. https://doi.org/10.1097/00004728-198701000-00016

[11] Van Randen, A., Lameris, W., van Es, H.W., van Heesewijk, H.P., van Ramshorst, B., Ten Hove, W., Bouma, W.H., et al. (2011) A Comparison of the Accuracy of Ultrasound and Computed Tomography in Common Diagnoses Causing Acute Ab- 
dominal Pain. European Radiology, 21, 1535-1545.

https://doi.org/10.1007/s00330-011-2087-5

[12] Hwang, H., Marsh, I. and Doyle, J. (2014) Does Ultrasonography Accurately Diagnose Acute Cholecystitis? Improving Diagnostic Accuracy Based on a Review at a Regional Hospital. Canadian Journal of Surgery, 57, 162-168. https://doi.org/10.1503/cjs.027312

[13] Fagenholz, P.J., Fuentes, E., Kaafarani, H., Cropano, C., King, D., de Moya, M., et al. (2015) Computed Tomography Is More Sensitive than Ultrasound for the Diagnosis of Acute Cholecystitis. Surgical Infections, 16, 509-512. https://doi.org/10.1089/sur.2015.102

[14] Barie, P.S. (2001) Acute Acalculous Cholecystitis in Surgical Treatment: Evidence-Based and Problem-Oriented. Zuckschwerdt, Munich.

[15] Iyer, S.G., Ravishankar, K.D., Huang, E. and Masud, K. (2007) Acute Acalculous Cholecystitis: Challenging the Myths. HPB (Oxford), 9, 131-134.

https://doi.org/10.1080/13651820701315307

[16] Pinto, A., Reginelli, A., Cagini, L., Coppolino, F., Ianora, A.A.S., Bracale, R., Giganti, M. and Romano, L. (2013) Accuracy of Ultrasonography in the Diagnosis of Acute Calculous Cholecystitis: Review of the Literature. Critical Ultrasound, 5, S11. https://doi.org/10.1186/2036-7902-5-S1-S11

[17] Van Breda Vriesman, A.C., Engelbrecht, M.R., Smithuis, R.H.M. and Puylaert, J.B.C.M. (2007) Diffuse Gallbladder Wall Thickening: Differential Diagnosis. American Journal of Roentgenology, 188, 495-501.

https://doi.org/10.2214/AJR.05.1712 